\title{
Loss of CEP70 function affects acrosome biogenesis and flagella formation during spermiogenesis
}

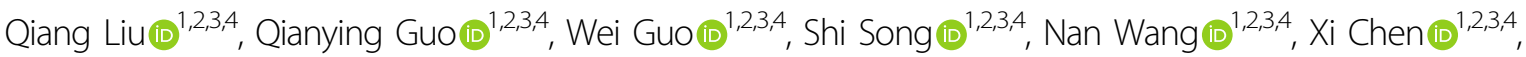 \\ Andi Sun ${ }^{1,2,3,4}$, Liying Yan ${ }^{1,2,3,4}$ and Jie Qiao (1) 1,2,3,4,5
}

\begin{abstract}
The spermatogenesis process is complex and delicate, and any error in a step may cause spermatogenesis arrest and even male infertility. According to our previous transcriptomic data, CEP70 is highly expressed throughout various stages of human spermatogenesis, especially during the meiosis and deformation stages. CEP70 is present in sperm tails and that it exists in centrosomes as revealed by human centrosome proteomics. However, the specific mechanism of this protein in spermatogenesis is still unknown. In this study, we found a heterozygous site of the same mutation on CEP70 through mutation screening of patients with clinical azoospermia. To further verify, we deleted CEP70 in mice and found that it caused abnormal spermatogenesis, leading to male sterility. We found that the knockout of CEP70 did not affect the prophase of meiosis I, but led to male germ-cell apoptosis and abnormal spermiogenesis. By transmission electron microscopy (TEM) and scanning electron microscopy (SEM) analysis, we found that the deletion of CEP70 resulted in the abnormal formation of flagella and acrosomes during spermiogenesis. Tandem mass tag (TMT)-labeled quantitative proteomic analysis revealed that the absence of CEP70 led to a significant decrease in the proteins associated with the formation of the flagella, head, and acrosome of sperm, and the microtubule cytoskeleton. Taken together, our results show that CEP70 is essential for acrosome biogenesis and flagella formation during spermiogenesis.
\end{abstract}

\section{Introduction}

Infertility is a serious health problem worldwide. According to the latest data from the World Health Organization (WHO), around $10-15 \%$ of couples (50-80 million people) of reproductive age are suffering from infertility. Male factors account for about $50 \%$ of these cases of infertility ${ }^{1-3}$. In addition, recent data showed that the male etiology of $\sim 70 \%$ of infertile couples remains

\footnotetext{
Correspondence: Liying Yan (yanliyingkind@aliyun.com) or Jie Qiao (jie.qiao@263.net)

${ }^{1}$ Center for Reproductive Medicine, Department of Obstetrics and Gynecology, Peking University Third Hospital, Beijing, China

${ }^{2}$ National Clinical Research Center for Obstetrics and Gynecology (Peking University Third Hospital), Beijing, China

Full list of author information is available at the end of the article

These authors contributed equally: Qiang Liu, Qianying Guo

Edited by A. Stephanou
}

unexplained ${ }^{4}$. In male infertility patients, there is a type of disease called oligoasthenoteratozoospermia (OAT) that is mainly characterized by a decreased sperm concentration and motility, and a higher rate of abnormal shapes ${ }^{5}$. As a key cause of male infertility, azoospermia or OAT is mainly caused by severe damage to spermatogenesis ${ }^{6}$. Spermatogenesis is a complicated process that involves the proliferation of spermatogonial stem cells (SSCs), meiosis of spermatocytes, haploid differentiation, and spermiogenesis ${ }^{7,8}$. In the process of spermiogenesis, round spermatids undergo a series of dynamic morphological changes, including the formation of acrosomes, mitochondrial sheath and flagella, and cytoplasmic clearance $^{9-11}$. The transcriptomic analyses have shown that there are more than 4000 genes involved in the process of human spermatogenesis ${ }^{12}$. Therefore, many

\section{(c) The Author(s) 2021}

(c) (i) Open Access This article is licensed under a Creative Commons Attribution 4.0 International License, which permits use, sharing, adaptation, distribution and reproduction c. in any medium or format, as long as you give appropriate credit to the original author(s) and the source, provide a link to the Creative Commons license, and indicate if changes were made. The images or other third party material in this article are included in the article's Creative Commons license, unless indicated otherwise in a credit line to the material. If material is not included in the article's Creative Commons license and your intended use is not permitted by statutory regulation or exceeds the permitted use, you will need to obtain permission directly from the copyright holder. To view a copy of this license, visit http://creativecommons.org/licenses/by/4.0/. 
unknown genetic factors that affect spermatogenesis require further investigation.

According to our previous transcriptomic data, CEP70, which belongs to the centrosome protein family (CEP family), is highly expressed throughout various stages of human spermatogenesis, especially during the meiosis and deformation stages ${ }^{13}$. Additionally, analysis of the reported proteomic data of human sperm tails found that CEP70 is present in sperm tails ${ }^{14}$. As a centrosome protein, CEP70, which was first discovered in human centrosome proteomics, is located in the centrosome throughout the cell cycle and interacts with $\gamma$-tubulin by two coiled-coil domains ${ }^{15,16}$. Within the CEP family, studies have shown that CEP135 and CEP131 are involved in spermatogenesis ${ }^{17,18}$. Moreover, CEP70 participates in the extension and dynamic regulation of microtubules and interacts with histone deacetylase 6 (HDAC6) to regulate the stability of microtubules ${ }^{19,20}$. In zebrafish, knocking down cep70 and cep131 can cause abnormal cilia production $^{21}$. Based on these data, we hypothesize that CEP70 may play important roles in regulating mammalian spermatogenesis and its mutation is related to male infertility.

In this study, we conducted mutation screening of patients with clinical azoospermia and found a heterozygous site of the same mutation on CEP70. To further verify, we deleted CEP70 in mice and found that it caused abnormal spermiogenesis, leading to male sterility. Finally, we used quantitative proteomic analysis to revealed that the absence of CEP70 led to a significant decrease in the proteins associated with the formation of the flagella, head, and acrosome of sperm, and the microtubule cytoskeleton.

\section{Results \\ CEP70 may play an important function in human spermatogenesis}

According to the single-cell transcriptome data of germ cells $^{13}$, CEP70 is highly expressed in various stages of human spermatogenesis, especially during the meiosis and deformation stages (Fig. 1A). To determine whether the CEP7O mutation is associated with clinical azoospermia patients, we designed 14 pairs of primers (listed in Supplementary Table S2) for the coding sequence of CEP70 to investigate the variation of it in 476 infertile patients with azoospermia and 252 men with normal fertility. After sequencing, we found that four subjects with azoospermia had the same heterozygous mutation site (c.269A $>$ T, p. N33I), while there was no such mutation in the control group (Fig. 1B). This mutation was conserved among various species (Fig. 1C) and predicted to be deleterious and possibly damaging by SIFT and PolyPhen2 (https://asia.ensembl.org/Multi/Tools/VEP). Through the clinical pathology report, it was found that two of the four cases had Sertoli cell-only syndrome (SCO), which caused azoospermia (Fig. 1D). However, there were no homozygous mutations or compound heterozygous mutations were found in the azoospermia
$\mathbf{A}$

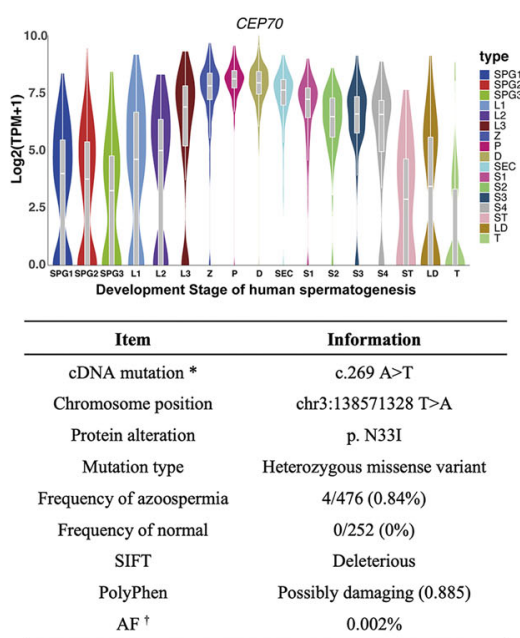

C

Zebrafish NP 001108048.1 ------MEQEEQREWDAVNRLLQHHG .... EDLMLIVDTLLDETQ Mouse NP_076362.2 SNRLMIEKOOEEAEWESINGLLMTHG $\cdots$ GDLLFMVDTMLEEVE Cattle XP_024851957.1 OIRLMTEKOREEAEWENINVLLMMHG $\cdots$ EDLLFIVDTMLEEVE Rabbit XP017202297.1 SDKLMAEKOOEEAEWENINMLIMKHG .... GDLLFMVDTMLEEIE Pig XP 013837515.2 STSVMTEKOREEEEWESTNVLLMTHG ... EDLLFMVDTMLEELE Human NP 001307527.1 SDRTMTEKOOEFAEVESTNVITMMHG .... EDLLFIVDTMLEEVE Ho Monkey NP_001253676.1 SDRLMTEKOOLEAENESINVLLMMHG .... EDLLFIVDTMLEEVE Horse XP_-
Dog XP_023476778.1
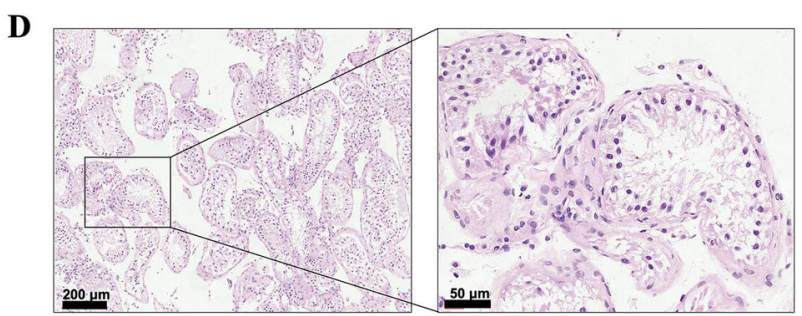

Fig. 1 The expression pattern of CEP70 during human spermatogenesis and clinical mutation screening. A The mRNA expression pattern of CEP70 during human spermatogenesis. SPG1: spermatogonial stem cells; SPG2: differentiating spermatogonia; SPG3: differentiated spermatogonia; L1-L3: three consecutive stages of leptotene spermatocytes; Z: zygotene; P: pachytene; D: diplotene; SEC: a mixture of diakinesis, metaphase, anaphase, telophase and secondary spermatocytes; S1-S4: four stages of spermatids; ST: Sertoli cell; LD: peritubular myoid cells (PMCs) and Leydig cells; T: testicular macrophages. B Analysis of CEP70 mutation in azoospermia patients. *: the accession number for CEP70 is GenBank: NM_024491.4; t: frequency of existing variant in 1000 Genomes combined population. $\mathbf{C}$ Homology analysis of amino acid changes caused by mutation sites among different species. D H\&E staining of clinicopathological sections of azoospermia patients with the same heterozygous mutation. 
patients, indicating that the recessive CEP70 may just relate to azoospermia and the deleterious heterozygous mutation of CEP70 may increase the risk of azoospermia.

\section{Knockout of Cep70 causes spermatogenesis disorders and male infertility}

A previous study of single-cell transcriptome data during spermatogenesis showed that Cep70 mRNA was highly expressed during the spermatogenesis of mice ${ }^{22}$ (Fig. 2A, up). Then, we sorted mouse spermatogenic cells at different stages by FACS and detected CEP70 protein expression in these samples by western blot. The results showed that CEP70 was highly expressed in spermatocytes and round spermatids (Fig. 2A, down).

To study the biological functions of CEP70, we used CRISPR/Cas9 technology to knockout the gene in mice (Fig. 2B). We obtained a mouse strain with two nucleotides inserted in exon five of the Cep70 gene, which is located downstream of the translation start site. This insertion caused a reading frameshift and created a
A

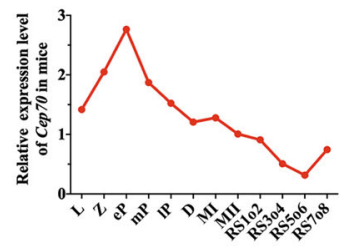

RS MII LZ PD

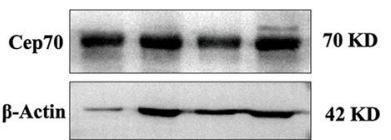

B

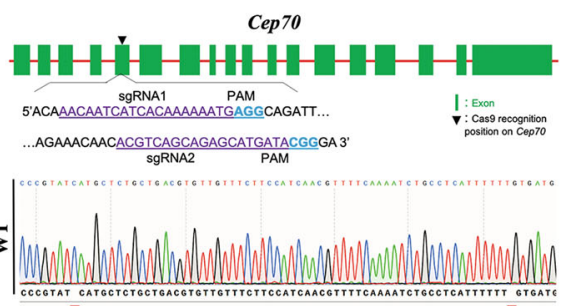

C

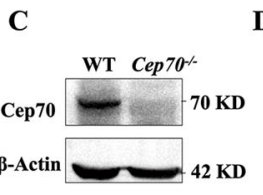

D

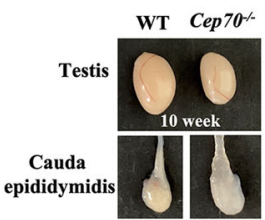

$\mathbf{E}$

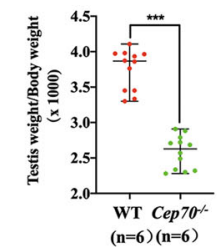

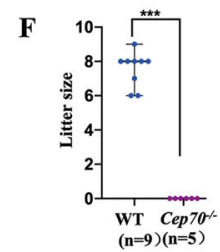

G

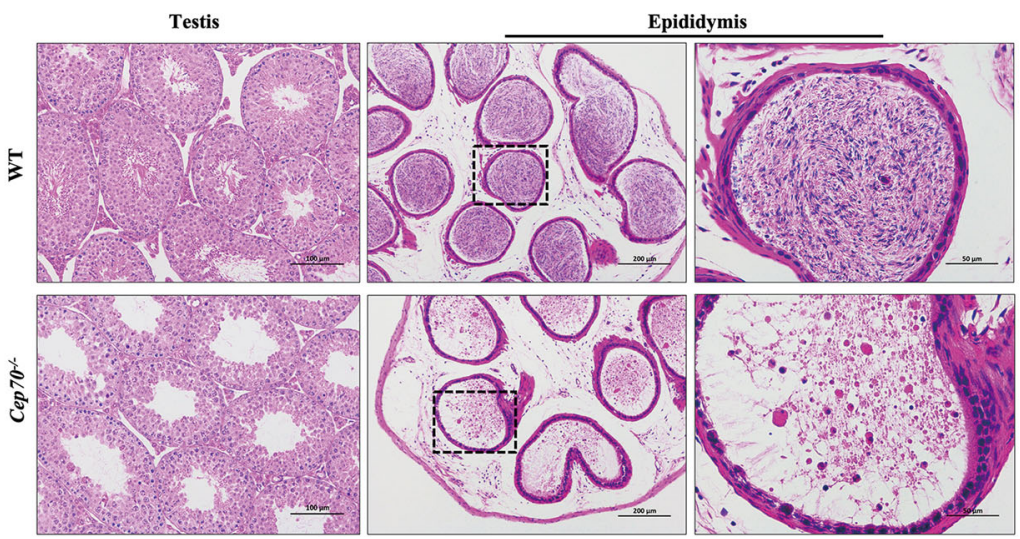

Fig. 2 Cep70 knockout caused male mice infertility. A (Up) Relative mRNA levels of Cep70 in mouse spermatocytes. L: leptotene; Z: zygotene; eP: early pachytene; mP: middle pachytene; IP: late pachytene; D: diplotene; Ml: metaphase I; MII: metaphase II; RS102: steps 1-2 spermatids; RS304: steps 3-4 spermatids; RS506: steps 5-6 spermatids; RS708: steps 7-8 spermatids. (Down) The expression pattern of protein and mRNA of CEP70 at different staged germ cells isolated by FACS during spermatogenesis in mice. LZ: leptotene and zygotene stage; PD: pachytene and diplotene stage; MII: meiosis II spermatocytes; RS: round spermatids. B Schematic diagram of generating Cep70-knockout mouse model (Cep70 ${ }^{-1-}$ ) by CRISPR/ Cas9 system. The genotype analysis results by Sanger sequencing showed a frameshift variant $(+1 /+1 \mathrm{bp})$ of Cep $70^{-\prime-}$ mice. $\mathbf{C}$ Western blotting showed that CEP70 was deleted in 10-week-old Cep70 ${ }^{-1-}$ whole testis lysates compared to wild type. $\beta$-Actin was used as the loading control. D Representative images of testis and cauda epididymidis from WT and Cep $70^{-1-}$ mice. The testis of Cep $70^{-1-}$ was smaller than that of the WT, the cauda epididymis of Cep70 ${ }^{-1-}$ was more transparent than that of the WT. (E) Testis weight to body weight ratio of WT and Cep70 ${ }^{-1-}$ mice at 10weeks $(n=6)$. Data are presented as the mean \pm SEM. $p<0.05\left(^{*}\right), 0.01\left(^{* *}\right)$, or $0.001\left(^{(* *)}\right.$. F Number of pups per litter from male mice $(>8$-weeks old) naturally crossed with WT female mice ( $>6$-weeks old) for 6 months. Cep $70^{-1-}$ male mice showed complete sterility. Data are presented as the mean $\pm \mathrm{SD}, n=5, p<0.001{ }^{(* * *)}$. G Histological analysis of the testis and epididymis seminiferous tubules from the WT and Cep70-1- mice. No elongated spermatids are present in Cep70 ${ }^{-1-}$ mice. Scale bar: $100 \mu \mathrm{m}$ (left); $200 \mu \mathrm{m}$ (middle); $50 \mu \mathrm{m}$ (right). 

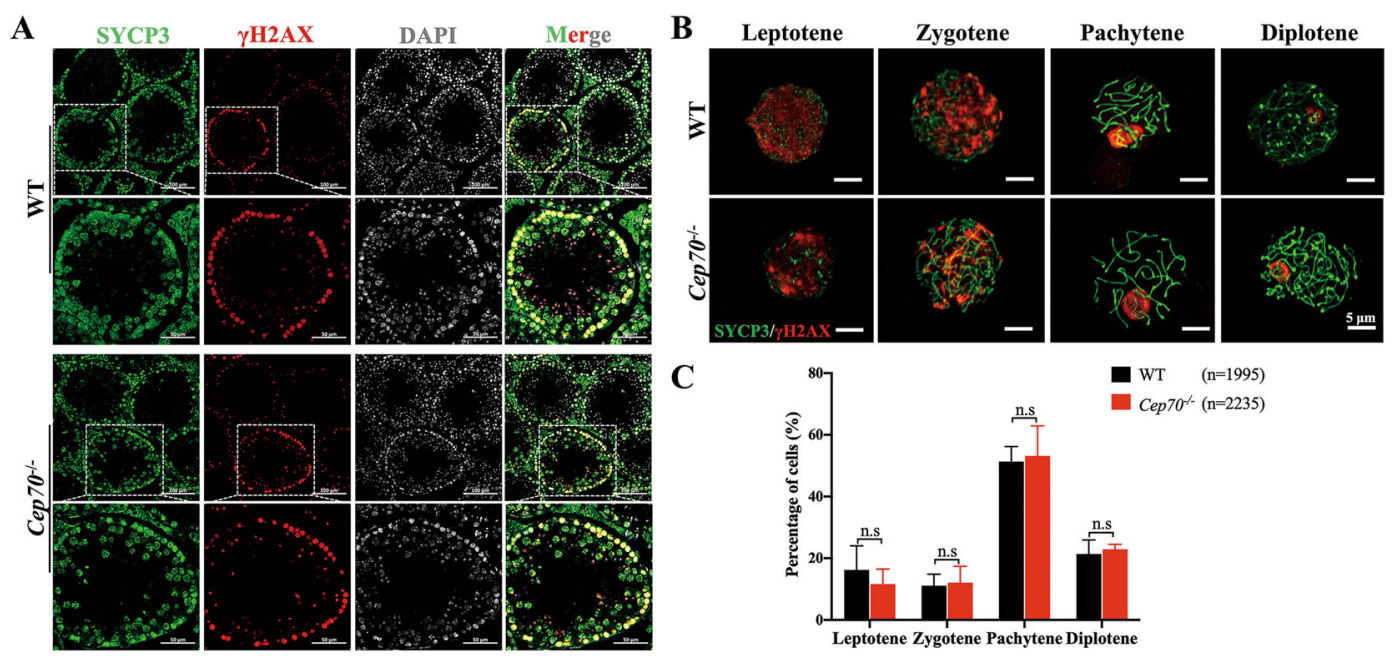

Fig. 3 CEP70 is dispensable to the prophase of meiosis I during spermatogenesis. A Immunofluorescence staining of SYCP3 (green) and $\gamma \mathrm{H} 2 \mathrm{AX}$ (red) in sections of testes from the WT and Cep $70^{-1-}$ mice. DAPI indicates the nucleus. Scale bar: $100 \mu \mathrm{m}$ (up), $50 \mu \mathrm{m}$ (down). B Immunofluorescence staining of SYCP3 (green) and YH2AX (red) in chromosome spreads of spermatocytes from the testes of 10-week-old WT and Cep70-1- male mice. Scale bar: $5 \mu \mathrm{m}$. C Quantification of the proportion of meiotic stages in WT and Cep70 ${ }^{-1-}$ spermatocytes. The numbers marked in the bars represent the percentage of cells at indicated meiosis stage (leptotene, zygotene, pachytene, and diplotene). For each genotype, three mice were analyzed. $p$-values were calculated by Student's t-test. Data are presented as the mean $\pm \mathrm{SD}$, n.s. not statistically significant.

premature stop codon (Fig. 2B). The deletion of CEP70 was detected by Western blot, and the CEP70 band was only detected in WT testis lysate (Fig. 2C), indicating that the CEP70 protein was successfully depleted in Cep70 (KO) mice. The growth and healthy of $\mathrm{Cep} 70^{-1-}$ mice were normal, and the Cep $70^{-1-}$ females have normal fertility. However, Cep $70^{-1-}$ males were completely sterile. Compared to WT male mice, the testis size of Cep $70^{-1-}$ male mice was small, and the testes weight was significantly reduced $(107.83 \pm 5.36 \mathrm{mg}$ versus $68.92 \pm$ $8.45 \mathrm{mg}$ ), as shown in Fig. 2D, E. In a 6-months fertility test, the $\mathrm{Cep} 7 \mathrm{O}^{-1-}$ male mice that mated with WT females had no offspring (Fig. 2F). Histological examination showed that the seminiferous tubules of testes and the epididymis of $\mathrm{Cep} 7 \mathrm{O}^{-1-}$ male mice were devoid of elongated spermatids. However, both spermatocytes and round spermatids were present in the $\mathrm{Cep} 70^{-1-}$ male mice testes (Fig. 2G). These results suggest that CEP70 plays an essential role in spermatogenesis.

\section{CEP70 is dispensable to the prophase of meiosis I during spermatogenesis}

To determine whether CEP70 deletion affects the number of germ cells, including spermatocytes, we performed immunofluorescence staining of $\mathrm{MVH}$, a germcell marker, to characterize the first wave of spermatogenesis in mice at 9 days postpartum (dpp), $12 \mathrm{dpp}$, and $14 \mathrm{dpp}$ that represent the generation time of leptotene, zygotene, and pachytene spermatocytes, respectively ${ }^{23}$. The results indicated that the numbers of germ cells had no significant differences in the first wave of spermatogenesis between WT and Cep $70^{-1-}$ male mice, suggesting that the prophase of meiosis I was not affected in Cep70-deficient mice (Supplementary Fig. S1A-D). To further confirm this result, we identified different stages of meiotic prophase I by staining of the testes section for phosphorylated $\mathrm{H} 2 \mathrm{AX}(\gamma \mathrm{H} 2 \mathrm{AX})$, a marker of unrepaired DNA lesions and the sex body in pachynema, and SYCP3, a component of the synaptonemal complex. The results showed that all stages existed in the spermatocyte nuclei of WT and Cep $70^{-1-}$ mice testes (Fig. 3A). To determine whether the ratios of spermatocytes at leptotene, zygotene, pachytene, and diplotene stage were abnormal, we performed spermatocyte spreading of WT and Cep $70^{-1-}$ mice testicular tissues (Fig. 3B). The statistical results showed that the lack of CEP70 did not cause abnormal spermatocyte development at all four stages (Fig. 3C). Additionally, we examined the chromosomal synapsis process during the prophase of meiosis I by staining for SYCP3 and SYCP1, important components of the synaptonemal complex. We found that the signals of SYCP1 were similar between WT and Cep $70^{-1-}$ mice at pachytene stage (Supplementary Fig. S1E). These results demonstrated that CEP70 deletion had no effects on the prophase of meiosis I.

\section{CEP70 deletion results in germ-cell apoptosis and abnormal spermiogenesis}

Although the number of germ cells at $9 \mathrm{dpp}, 12 \mathrm{dpp}$, and $14 \mathrm{dpp}$ of the WT and Cep $70^{-1-}$ male mice testis were not affected, the results of MVH staining showed that the loss of CEP70 caused a significant decrease in the 


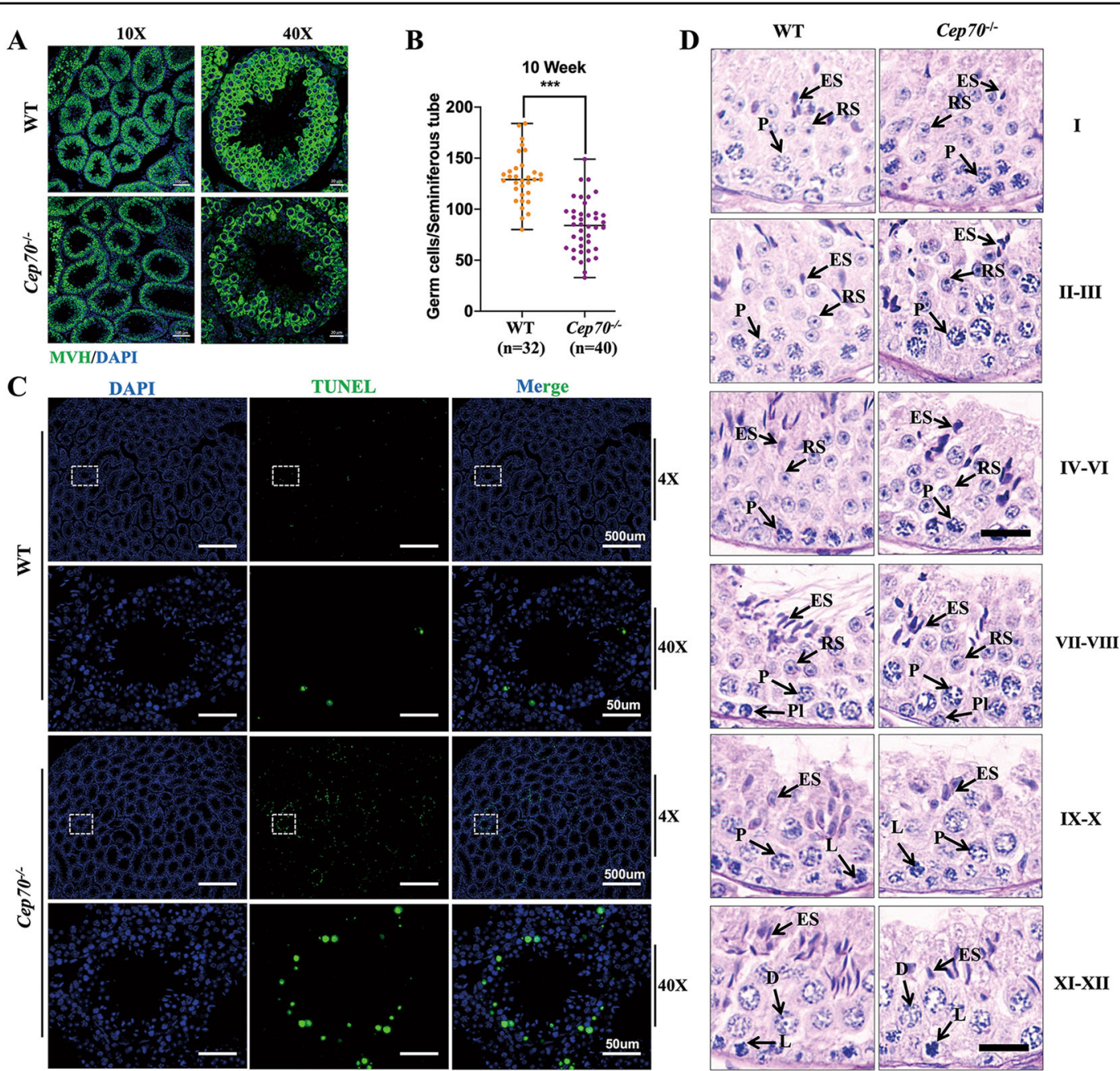

Fig. 4 Cep70-knockout mice showed germ-cell apoptosis and abnormal spermiogenesis. A MVH immunofluorescence staining of testes sections of the WT and Cep $70^{-1-}$ mice, demonstrating a significant decrease of germ cells in the seminiferous tubules of Cep $70^{-1-}$ mice. Scale bar: $10 \mu \mathrm{m}$ (left), $20 \mu \mathrm{m}$ (right). B Quantification of germ cells in each seminiferous tubule of the WT $(n=32)$ and Cep70 ${ }^{-1-}$ mice $(n=40)$ at 10-weeks of age. Data are presented as the mean $\left.\pm \mathrm{SD}, p<0.001{ }^{(* *}\right)$. C TUNEL immunofluorescence staining of testes sections of the WT and Cep70-1- mice. The TUNEL-positive signal (green) level was significantly higher in Cep70-1- mice. Scale bar: $500 \mu \mathrm{m}$ (up), $50 \mu \mathrm{m}$ (down). D PAS-hematoxylin staining of seminiferous tubules from the WT and Cep70 ${ }^{-1-}$ mice, indicating spermatogenic arrest at stages VII-VIII in the Cep $70^{-/-}$mice. Scale bar: $20 \mu \mathrm{m}$.

number of germ cells at 10-weeks (Fig. 4A, B), probably because the germ cells were undergoing apoptosis. The TUNEL assay results showed that the apoptosis level of germ cells was significantly increased in Cep $70^{-1-}$ mice testes (Fig. 4C). To identify exactly which stage of spermatogenesis was affected in Cep70-deficient mice, we performed PAS staining to distinguish the 12 stages (I-XII) of spermatogenesis. The results showed that spermatogenesis of Cep70-deficient mice was blocked at stages VII and VIII, and most of the round spermatids could not be transformed into elongated spermatids (Fig. 4D). According to previous reports, Sertoli cells play an important role in the stage of spermiogenesis ${ }^{24}$. In order to determine whether Cep70 deletion affects the development and localization of Sertoli cells, SOX9 (a marker of Sertoli cells) staining results showed that there was no difference in the number and location of Sertoli cells in the testes of WT and Cep $70^{-1-}$ male mice (fig. S2). Together, these findings suggest that the absence of CEP70 might lead to abnormal spermiogenesis in male mice and ultimately leads to infertility.

\section{Loss of CEP70 function results in abnormal formation of flagella and acrosomes}

To further determine whether there was an abnormality in the process of spermatid head shaping, SEM and TEM were performed. The results of SEM showed that the mature sperm of WT male mice had a complete structure, including obvious equatorial segments, a post-acrosomal sheath, a ventral spur, and a sharp hook rim (Fig. 5A). 

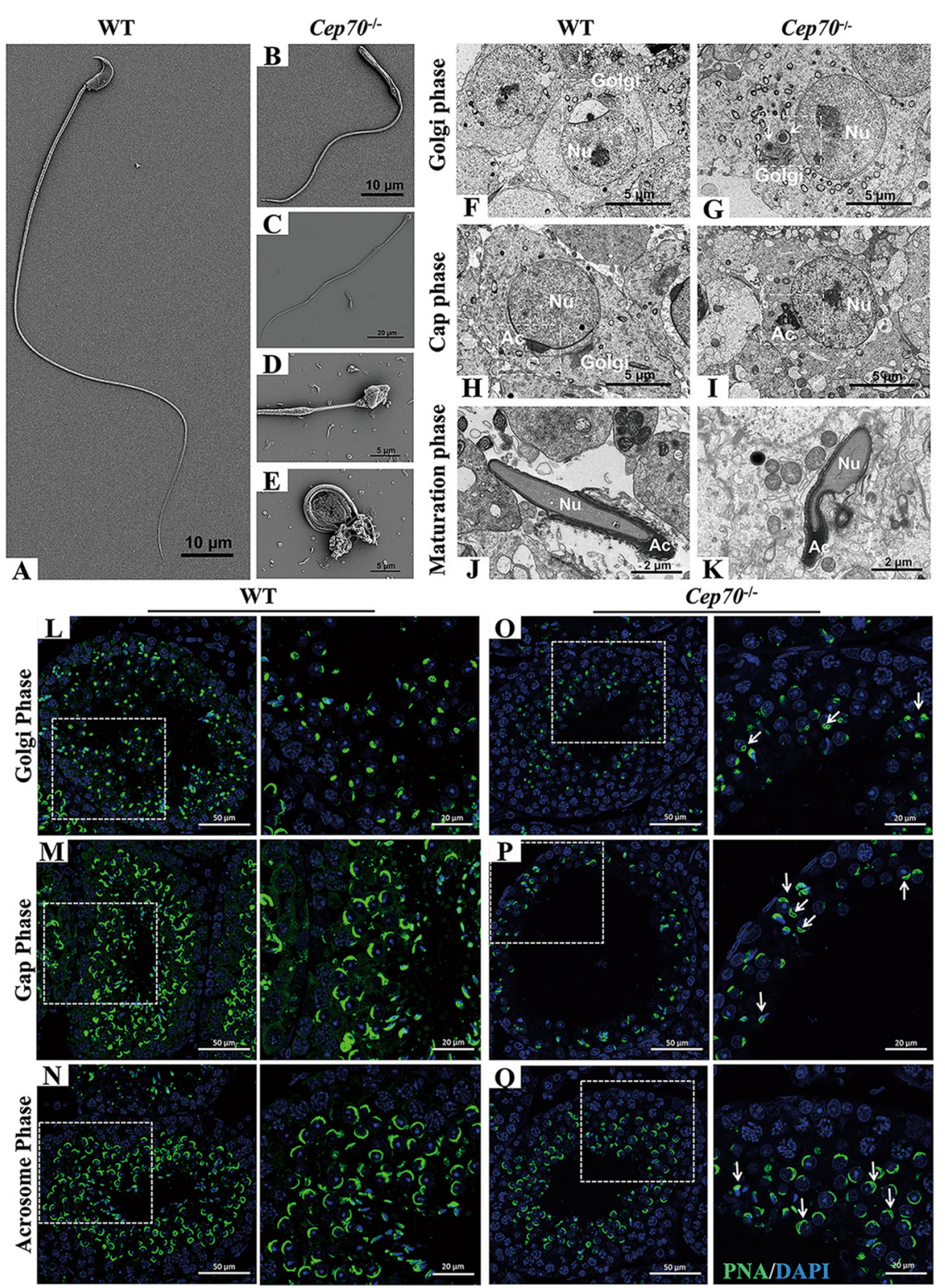

Fig. 5 Cep70-knockout mice showed the abnormal formation of flagella and acrosomes. A-E SEM analysis showed the morphology of sperm. Sperm with normal structure (A) was observed in the WT mice. Sperm with head defects, including irregular shape (B, D) and microcephaly (C), and tail deformities $(\mathbf{E})$ were observed in the Cep $70^{-1-}$ mice. $\mathbf{F}-\mathbf{K}$ TEM analysis demonstrated the Golgi phase $(\mathbf{F}, \mathbf{G})$, cap phase $(\mathbf{H}, \mathbf{I})$ and maturation phase $(\mathbf{J}, \mathbf{K})$ of the $\mathbf{W T}(\mathbf{F}, \mathbf{H}, \mathbf{J})$ and $\operatorname{Cep}^{-1-}(\mathbf{G}, \mathbf{I}, \mathbf{K})$ mice. Multiple acrosomal structures $(\mathbf{G})$, vacuolated acrosomes $(\mathbf{I})$, and irregularly shaped acrosomes (K) were observed in Cep70 ${ }^{-1-}$ mice. Nu: nucleus; Ac: acrosome. Scale bar: 5 um (F-I), $2 \mu \mathrm{m}(\mathbf{J}-\mathbf{K})$. L-Q Immunofluorescence staining with FITC-conjugated PNA (green) on sections of the WT $(\mathbf{L}-\mathbf{N})$, and $\operatorname{Cep}^{-1-}(\mathbf{O}-\mathbf{Q})$ mice testes, including Golgi phase (L, $\left.\mathbf{O}\right)$, gap phase $(\mathbf{M}, \mathbf{P})$, and acrosome phase $(\mathbf{N}, \mathbf{Q})$. Images displayed on the right, show the higher magnification of the boxed areas. Arrows indicate defective acrosome structures. Scale bar: $50 \mu \mathrm{m}$ (left), $20 \mu \mathrm{m}$ (right).

Although there were spermatozoa with flagella in $\mathrm{Cep} 70^{-1-}$ mice epididymis, the tail and head were abnormal compared to WT mice (Fig. 5B-E). Sperm head defects showed microcephaly and irregular shapes, and tail deformities showed bending, coiling, wrinkling, and shortening. Furthermore, we found that the sperm head of Cep $70^{-1-}$ mice also had an abnormal acrosome structure, which suggested that the deletion of CEP70 might also affect the acrosome biogenesis. To verify this hypothesis, we conducted a TEM analysis of the testes. TEM results showed that the Golgi, cap and maturation phases in WT mice testes could be identified by their standard characteristics (Fig. 5F, H, J). However, multiple acrosomal structures could be found in the Golgi phase of Cep $70^{-1-}$ 
mice testes (Fig. 5G). Additionally, the vacuolated or irregularly shaped acrosomes were detected in the cap and maturation phases of $\mathrm{Cep} 70^{-1-}$ mice spermatids (Fig. 5I, $\mathrm{K})$. Moreover, we found that WT and $\mathrm{Cep} 70^{-1-}$ testis sperm flagellar cross-section (principal and end piece) of each structure were not different, according to the TEM analysis (Supplementary Fig. S3). This result demonstrated that the absence of CEP70 did not affect the ultrastructure of sperm flagellar.

To further confirm the defects in acrosome formation in Cep $70^{-1-}$ mice, we performed immunofluorescence staining on testis sections with FITC-conjugated peanut agglutinin (PNA), a marker of the outer acrosomal membrane of spermatids. As expected, the WT spermatocytes had normal acrosome at Golgi, cap and acrosome phases (Fig. 5L-N). However, the Cep70 null spermatocytes exhibited abnormal acrosome structures (e.g., multiple acrosome centers and discrete structures) at all three acrosomal differentiating phases (Fig. 5O-Q), which was similar to observations from TEM analysis. Together, these results indicated that the CEP70 deficient affected the process of acrosome biogenesis. In addition, we found that the total sperm, motile sperm and progressive sperm were significantly decreased by CASA (Supplementary Fig. S4). The data further indicate that CEP70 was involved in acrosome biogenesis and sperm flagellar formation and its loss can lead to OAT-like phenotypes and further cause male infertility.

\section{CEP70 regulates key proteins of acrosome and flagella formation in spermatogenesis}

To investigate the molecular mechanism of CEP70 in the development of acrosome biogenesis and sperm flagellar formation, quantitative proteomic analyses of testes of WT and Cep $70^{-1-}$ male mice were performed by TMT labeling, HPLC fractionation and LC-MS/MS analysis (Fig. 6A). The fold change (FC) in the amount of differential expression exceeding 1.3, and less than $1 / 1.3(\mathrm{KO} /$ WT) was regarded as the change threshold for a significant upregulation and downregulation, respectively. A total of 4608 proteins were quantified, of which 259 proteins were identified to be differentially expressed (Supplementary Table S3, $p<0.05$ ), including 167 downregulated and 92 upregulated proteins (Fig. 6B). GO annotation and enrichment analysis of DEPs in WT and Cep $70^{-1-}$ testes showed that 61 DEPs are related to the development of spermiogenesis (e.g., sperm part, acrosomal vesicle, motile cilium, sperm flagellum, $9+2$ motile cilium, cilium, sperm fibrous sheath, and ciliary part) among the downregulated proteins (Fig. 6C). The results of the PPI network indicated that most downregulated proteins interact with each other and play important functions in spermatogenesis. In addition, the absence of CEP70 also caused the upregulated the expression of proteins related to the biological processes of ribosome and protein digestion and absorption, indicating that these two biological processes may be involved in the regulation of spermatogenesis (Fig. 6D). Furthermore, the heatmap of 73 downregulated proteins displayed sharp differences between WT and Cep $70^{-1-}$ male testes and high-quality repeatability between samples in the same group (Fig. 6E).

In order to further verify the quality of the proteomic data, we further selected 15 proteins that play important regulatory functions in the process of spermatogenesis from the four terms based on the GO annotation results, including sperm flagellum [A-kinase anchoring protein 4 $(\text { AKAP4 })^{25}$, tektin-4 (Tekt 4$)^{26}$, outer dense fiber of sperm tails 1 (ODF1 $)^{27}$, calcium binding tyrosine phosphorylation regulated $(\mathrm{CABYR})^{28}$, Rhophilin associated tail protein 1 (ROPN1 $)^{29}$, and thioredoxin domain-containing protein 2 (TXNDC2 $)^{30,31}$ ], sperm head [A disintegrin and

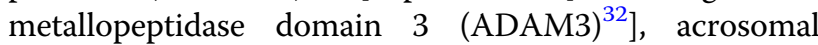
vesicle [A-kinase anchoring protein 3 (AKAP3) $^{33}$, Zona pellucida sperm-binding protein 3 receptor $(\mathrm{ZP} 3 \mathrm{R})^{34}$, sperm acrosome membrane-associated protein 1 $(\mathrm{SPACA} 1)^{35}$, Acrosin-binding protein (ACRBP) ${ }^{36}$, Zona pellucida-binding protein (ZPBP) ${ }^{37,38}$, and Izumo spermegg fusion protein 1 (IZUMO1) $\left.^{39,40}\right]$, and microtubule cytoskeleton [Protein Hook homolog 1 (HOOK1 $)^{41}$, Outer dense fiber of sperm tails 2 (ODF2) ${ }^{42}$ ], and performed PRM analysis on the testis protein samples of WT and Cep $70^{-1-}$ male mice (Supplementary Table S4) ${ }^{43}$. As shown in Fig. 7, the expression levels of all the 15 selected proteins were significantly decreased in $\mathrm{Cep} 70^{-1-}$ male mice compared with WT. Moreover, the fold difference of $\mathrm{KO} / \mathrm{WT}$ ratio was noticeably smaller in PRM than TMTlabel quantification. These results indicate that the CEP70 deficiency affects the proteins associated with acrosome and flagella formation during spermatogenesis.

\section{Discussion}

Our previous transcriptomic data demonstrated that CEP70 is highly expressed during meiosis and spermiogenesis, suggesting that CEP7O may play an indispensable function during spermatogenesis ${ }^{13}$. The human CEP7O is located at 3q22.3, with 18 exons that encodes a 597 amino acid protein $(\sim 70 \mathrm{kD})$, and the $\mathrm{N}$ terminus (1-326 amino acid) contains two coiled-coil domains, and the $\mathrm{C}$ terminus (327-597 amino acid) contains a tetratricopeptide repeat (TPR) domain according to the database of UniProt (https:// www.uniprot.org/uniprot/Q8NHQ1\#structure). In this study, we investigated the potential role and functional importance of CEP70 in the regulation of acrosome biogenesis and sperm flagellar formation during spermatogenesis by generating $\mathrm{Cep} 7 \mathrm{O}^{-1-}$ mice using CRISPR/Cas9 technology. Cep70-deficient male mice show complete infertility and severely impaired spermatogenesis. To the 


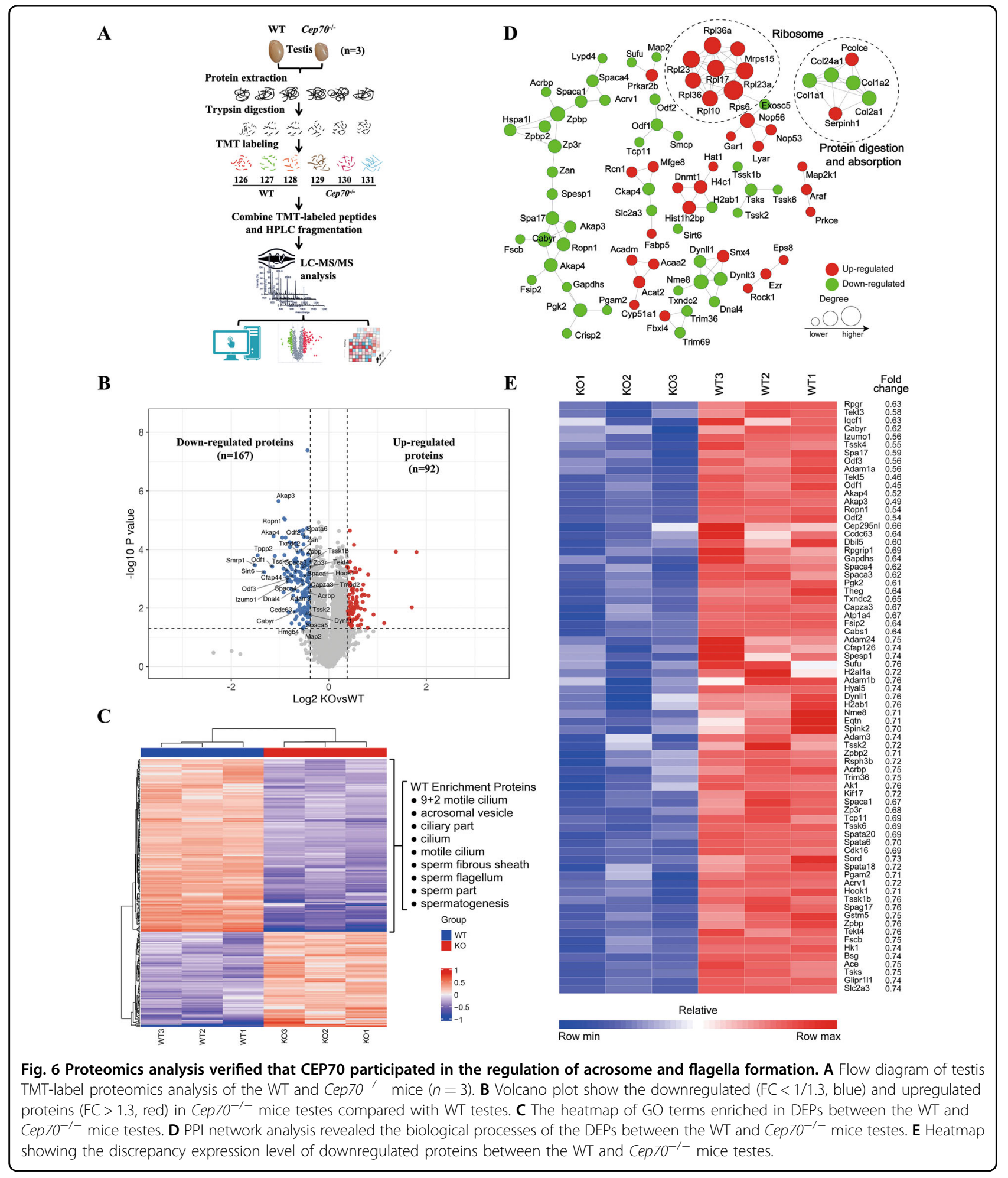

best of our knowledge, this is the first report on the involvement of CEP70 in the regulation of spermatogenesis.

By comparing and analyzing the exon sequences of CEP70 in azoospermia patients and normal males, we found that four subjects had the same heterozygous mutation site, which was predicted to be a deleterious mutation. Unfortunately, there were no homozygous mutations or compound heterozygous mutations were found in the azoospermia patients, indicating that the recessive CEP70 may just relate to azoospermia and the 

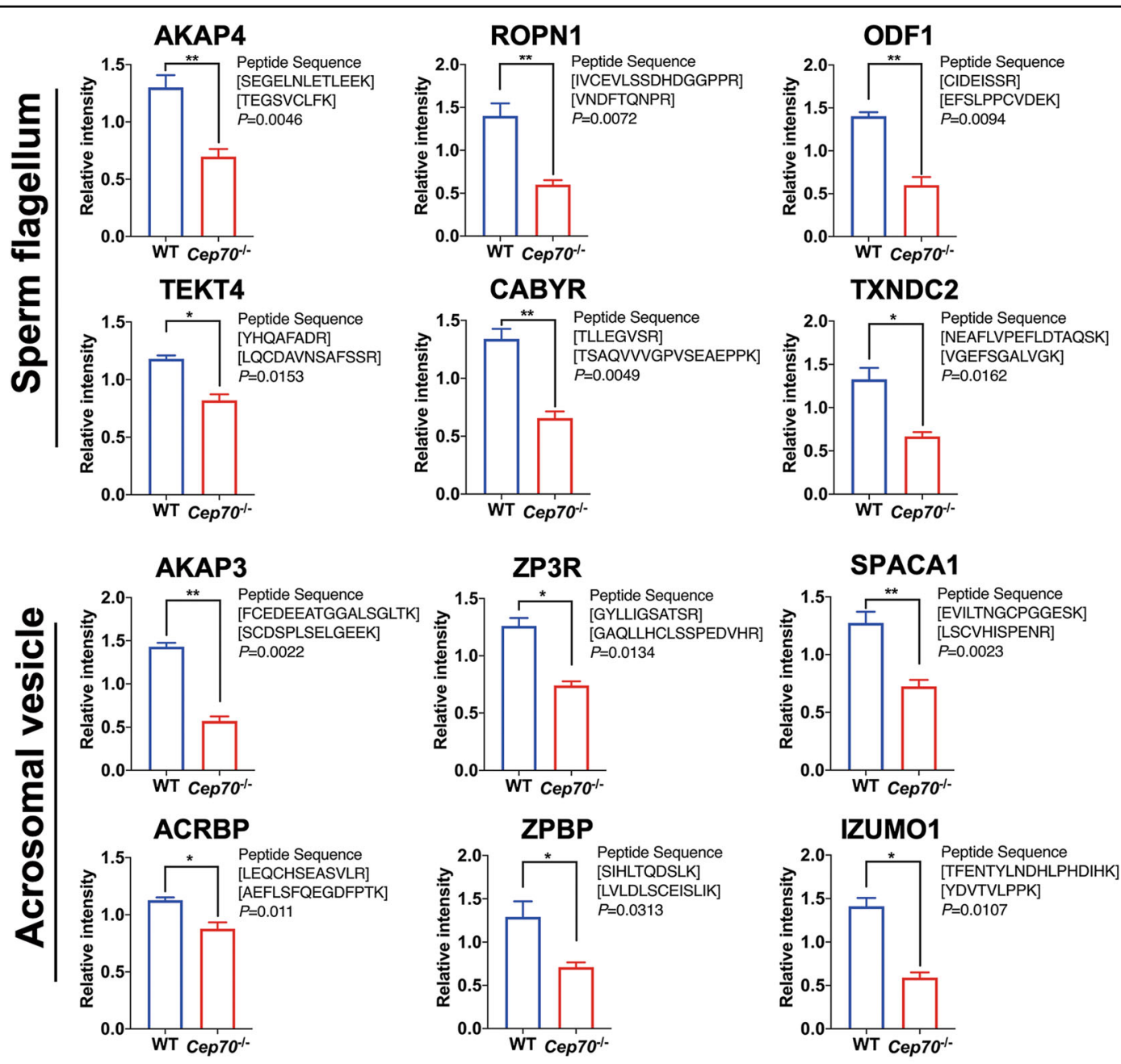

ADAM3

HOOK1
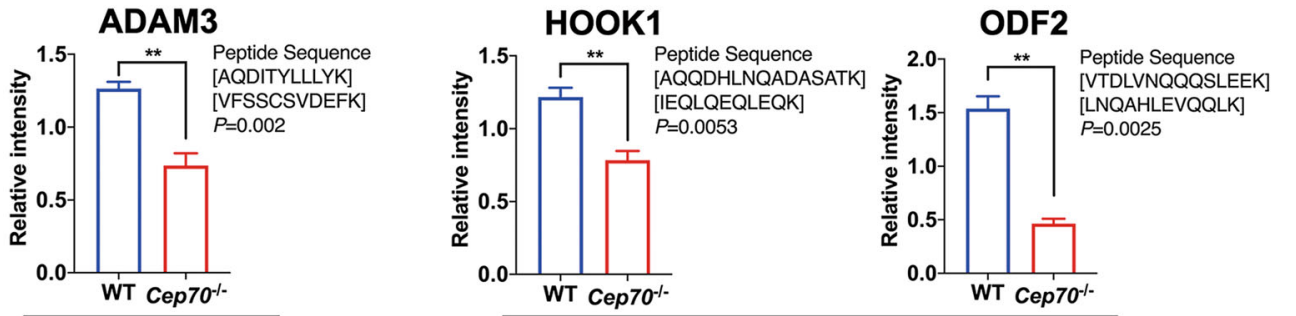

\section{Sperm head}

\section{Microtubule cytoskeleton}

Fig. 7 Fifteen proteins were validated in WT and Cep $70^{-1-}$ male mice testes by PRM. Fifteen downregulated proteins associated with sperm flagella, sperm head, acrosomal vesicle, and microtubule cytoskeleton, including AKAP4, ROPN1, ODF1, TEKT4, CABYR, TXNDC2, AKAP3, ZP3R, SPACA1, ACRBP, ZBPB, IZUMO1, ADAM3, HOOK1, and ODF2 showed significant differences in Cep70 ${ }^{-1-}$ male mice testes compared with WT testes ( $n=3$ ). Data are presented as the mean \pm SD. $p<0.05(*), 0.01\left(^{(*)}\right.$, or $0.001\left(^{(* *}\right)$.

deleterious heterozygous mutation of CEP70 may increase the risk of azoospermia. Therefore, we hypothesized that CEP70 is involved in the regulation of spermatogenesis, and used CRISPR/Cas9 technology to construct a global knockout model of Cep70 to study its biological functions. By analyzing the histological changes of the testis and epididymis, we found that CEP70 deficiency resulted in impaired spermatogenesis. Fertility testing revealed that the absence of CEP70 caused complete infertility in male mice.

Then, we found that the loss of CEP70 function caused the spermatogenesis to be blocked at stages VII and VIII, leading to no sperm flagella formation through PAS staining. The concentration of sperm in the epididymis 
and the number of motile sperm in $C e p 70^{-1-}$ mice was comparable to that in WT mice by CASA, and there was no motility sperm in the epididymis of $\mathrm{Cep} 70^{-1-}$ mice. The main manifestation of oligospermia is a significant decrease in the number of spermatids due to the apoptosis of germ cells or the failure of sperm release from the seminiferous epithelium ${ }^{44}$. The TUNEL assay showed that germ cells had obvious apoptosis in Cep $70^{-1-}$ mice. By comparing the testicular proteome data of WT and Cep $70^{-1-}$ mice, the loss of CEP70 caused a significant downregulation of the expression of DYNLL1, leading to the high expression of pro-apoptotic proteins that cause germ-cell apoptosis ${ }^{45,46}$. More remarkably, through SEM analysis we found that sperm lacking CEP70 had abnormal acrosomes and irregular tails; thus, the poor progressive motility in Cep $70^{-1-}$ spermatozoa could be attributed to the abnormal head and flagellar morphology. An earlier study showed that the zebrafish homolog of CEP70 can promote cilia assembly by controlling the length of the axoneme in zebrafish embryos ${ }^{21}$. Through this series of phenotypic analyses, we determined that the loss of CEP70 caused the OAT phenotype (low spermatozoa count, poor sperm motility, and abnormal sperm head structure) of male mice ${ }^{47,48}$. OAT, partially caused by defects in spermiogenesis, is the most common clinical profile in infertile men, including oligozoospermia, asthenozoospermia, and teratozoospermia ${ }^{49,50}$. However, non-obstructive azoospermia (NOA) is caused by a critical failure in the early stage of spermatogenesis (abnormal sperm self-renewal or meiotic arrest), which is different from OAT symptom ${ }^{49}$. Overall, we determined that CEP70 participates in the regulation of spermiogenesis, and the genetic alteration of Cep70 might related to OAT phenotype. According to the results of TEM and PNA immunofluorescence staining, CEP70 plays an important role in acrosome formation. Analysis of the proteomic data revealed that the components of the acrosome matrix (ZP3R and ZAN) in Cep $70^{-1-}$ mice were significantly downregulated compared to WT mice $^{51}$. After capacitation, ZP3R (also named SP56), a zona pellucida-binding protein, is located on the sperm surface and released at the time of the fusion of outer acrosomal and plasma membranes ${ }^{52}$. Additionally, the expression of another two membrane proteins (IZUMO1 and SPACA1) required for head shaping and oocyte fusion in $\mathrm{Cep} 70^{-1-}$ mice testes were significantly decreased compared to WT testes ${ }^{53,54}$. Therefore, CEP70 plays an important regulatory function in the process of acrosome formation.

Additionally, 61 downregulated proteins associated with the formation of sperm tail according to $\mathrm{GO}$ annotation were identified in the Cep $70^{-/-}$mice compared with the WT mice. Among them, it has been reported that the deletion of some genes (Spata6, Ropn1, and Cabyr) in a mouse model showed abnormalities and deformities of the sperm tail structure ${ }^{55}$. Studies have shown that the ROPN1 and CABYR expression level decreased remarkably in asthenospermia, and they can bind to AKAP3 to regulate the PKA signaling pathway and affect sperm flagella formation ${ }^{28,56}$. The depletion of spermatogenesis associated 6 (SPATA6) disrupts the proper formation of the sperm connecting piece and head-tail conjunction ${ }^{57}$. Furthermore, testis-specific serine/threonine-protein kinase 1, 2, and 4 (TSSK1, 2, and 4) regulate the development of sperm flagellum ${ }^{58,59}$. In our analysis of the proteomics data, it was found that the expression of ROPN1, CABYR, SPATA6, TSSK1, TSSK2, TSSK4, and AKAP3 decreased significantly after CEP70 deficiency, so the sperm of $\mathrm{Cep} 7 \mathrm{O}^{-1-}$ mice showed a phenotype of flagella formation failure. Moreover, the mutation of CAPZA3 can cause male infertility and show an OAT-like phenotype, which may also be one of the important reasons for the OAT phenotype caused by CEP70 deletion ${ }^{60}$.

In summary, our genetic and functional data, based on human subjects and mouse models, strongly suggest that heterozygous deleterious mutations of CEP70 may be a novel genetic cause of OAT. Additionally, the underlying mechanism that CEP70 regulates sperm flagellar development was elucidated in this study. The screening of the deleterious mutations of CEP7O could be important for clinical molecular diagnosis of male infertility. As the findings about CEP70 in this study are novel, further research is required on this unfamiliar gene to explore the importance of CEP7O in male fertility.

\section{Materials and methods}

\section{Mutation screening and sanger sequencing}

To scan for CEP7O mutations in infertile male patients, we used DNA samples collected from 476 infertile patients with azoospermia and 252 men with normal fertility, from the our reproductive Center between 2013 and 2016 to perform CEP7O exon amplification. The collection of these samples was approved in 2012 by the Reproductive Medicine Ethics Committee of Peking University Third Hospital (Item number: 2012sz018). The OneTaq ${ }^{\circledR}$ Hot Start QuickLoad ${ }^{\circledR} 2 X$ Master Mix (New England Biolabs, M0488S) was used for PCR amplification, and the 14 coding exon sequences of CEP70 were analyzed by Sanger sequencing using an ABI 3730xl DNA sequencer (Applied Biosystems $^{\mathrm{TM}}$ ) by BeiJing Ruibio Biotech Co., Ltd. The sequencing results were compared with the reference genome sequence of CEP70. The primers used for the PCR are listed in Supplementary Table S2.

\section{Generation of the Cep70-knockout mouse model}

According to experimental procedures similar to those reported previously ${ }^{61,62}$, a Cep70 (NCBI: NM_023873.4) 
knockout mouse model (termed $\mathrm{Cep} 70^{-/-}$) with the frameshift variant was obtained using the CRISPR/ Cas9 system, as shown in Fig. 1A. Two pairs of singleguide RNAs (sgRNAs) were designed for Cep70 on exon five. Sanger sequencing was used to identify the genotype of the founder mouse and its offspring with a frameshift variant. The sequence information of the sgRNAs and genotyping primers are listed in Supplementary Table S1. Mice, with a C57BL/6J genetic background, were maintained under controlled temperature $\left(20-22^{\circ} \mathrm{C}\right)$, appropriate humidity (50-70\%), lighting conditions (12/12 h light/dark cycle), and food and water provided ad libitum. The Cep70-knockout mice used in the experiment were 8-weeks of age or older. All experimental procedures and animal care were approved by the Animal Care and Use Committee of the Peking University Health Science Center. During the experiment, the mice in the WT group and the $\mathrm{KO}$ group were randomly selected without any subjective factors, and the number of animal samples in each experiment was guaranteed to be at least 3 or more.

\section{Fertility assessment of mice}

To assess the fertility of the Cep $70^{-1-}$ mice, sexually mature male ( $>8$-weeks old, $n=5$ ) and female ( $>6$-weeks old, $n=5)$ Cep $70^{-1-}$ mice were mated with two wild-type (WT) female ( $>6$-weeks old) and male (>8-weeks old) mice for 6 months. During this period, the two WT female mice were replaced every other gestation cycle. Meanwhile, WT female ( $>6$-weeks old) and male mice ( $>8$-weeks old) were mated 1:2, as the control group ( $n=$ $5)$. Litter sizes were recorded during the fertility assessment of Cep $70^{-1-}$ and WT mice.

\section{Histological and immunofluorescence analyses}

For histological examination, testes and epididymis were obtained from 10-week old Cep $70^{-1-}$ and WT males, immediately fixed in modified Bouin's fixative or $4 \%$ paraformaldehyde (PFA) for $24 \mathrm{~h}$ and stored in $75 \%$ ethanol. Periodic acid-Schiff (PAS) and hematoxylin-eosin (H\&E) staining were performed on formalin-fixed, paraffin-embedded testis, and epididymis sections $(3-4 \mu \mathrm{m})$. The PAS dye solution set was used for PAS staining (Servicebio, G1008), according to manufacturer instructions.

For immunofluorescence analysis, the slices were deparaffinized with xylene and alcohol, retrieved in ethylenediaminetetraacetic acid (EDTA) antigen retrieval buffer ( $\mathrm{pH} 8.0$ or 9.0) and placed at room temperature to cool down. Then, the tissue sections were blocked with $3 \%$ bovine serum albumin (BSA) for $1 \mathrm{~h}$, and incubated overnight at $4{ }^{\circ} \mathrm{C}$ with the following antibodies: anti-MVH (Abcam, ab13840, 1:500, rabbit), anti-SYCP3 (Abcam, ab97672, 1:100, mouse), anti- $\gamma \mathrm{H} 2 \mathrm{AX}$ (Abcam, ab11174, 1:400, rabbit), anti-SOX9 (Novus Biologicals, NBP1-
85551, 1:100, rabbit), and lectin PNA with Alexa Fluor ${ }^{\mathrm{TM}}$ 488 Conjugate (ThermoFisher, L21409, 1:200). The slides were then washed three times with phosphate-buffered saline (PBS) and incubated for $1 \mathrm{~h}$ with goat anti-rabbit Alexa Fluor ${ }^{\mathrm{TM}} 488$ - or 594-conjugated secondary antibody (Invitrogen, A32766). After washing three times with PBS, the slides were mounted with ProLong ${ }^{\mathrm{TM}}$ Gold Antifade Mountant with 4',6-diamidino-2-phenylindole (DAPI) (Invitrogen, P36931) and then imaged using a Zeiss LSM880 confocal microscope.

\section{Meiotic nuclear spreading and immunofluorescence staining}

Testes were collected from adult male mice, and spermatocyte surface spreading were made as previously reported $^{63}$. In brief, seminiferous tubules were treated with hypotonic extraction buffer (HEB, $\mathrm{pH}$ 8.2) for 30-60 min, and then pulverized in $100 \mathrm{mM}$ sucrose buffer (pH 8.2) and gently pipetted to form a cell suspension. The suspension was loaded onto adhesive slides containing $1 \%$ PFA and $0.15 \%$ Triton X-100 fixative (pH 9.2). After $2 \mathrm{~h}$ of incubation, the slides were air-dried overnight. Finally, the slides were washed with PBS (pH 7.4) three times ( 5 min each) and immunostained with primary antibodies, including anti-SYCP3 (a gift from Hengyu Fan lab, 1:400, rat), anti-SYCP1 (Abcam, ab15090, $1: 200$, rabbit), and anti- $\gamma \mathrm{H} 2 \mathrm{AX}$ (Abcam, ab11174, 1:400, rabbit), according to the above immunofluorescence protocol.

\section{Isolation of spermatogenic cells}

To isolate different types of spermatogenic cells (LZ: leptotene/zygotene spermatocytes; PD: pachytene/diplotene spermatocytes; MII: meiosis II spermatocytes; and RS: round spermatids), we performed fluorescenceactivated cell sorting (FACS) using a Flow Cytometer ARIA II (BD Biosciences), as previously reported ${ }^{22}$. Briefly, testes of 10 -week-old WT and Cep $70^{-1-}$ males mice were incubated in $5 \mathrm{~mL}$ Hank's Balanced Salt Solution (HBSS) with collagenase type I $(100 \mathrm{U} / \mathrm{mL}$, Gibco, 17100-017) after removal of the tunica albuginea at $34^{\circ} \mathrm{C}$ for $10 \mathrm{~min}$. The digested testes were further digested with $5 \mathrm{~mL}$ trypsin $(0.25 \%)$ containing $100 \mu \mathrm{L}$ DNase I ( $5 \mathrm{mg} /$ $\mathrm{mL}$ ) at $34^{\circ} \mathrm{C}$ for $8 \mathrm{~min}$, and then terminated by adding $500 \mu \mathrm{L}$ fetal bovine serum (FBS). The cell suspension was filtered through a $70 \mu \mathrm{m}$ cellular filter and centrifuged, then resuspended in Dulbecco's Modified Eagle Medium (DMEM) with Hoechst 33342 (Invitrogen, H3570, $10 \mathrm{mg} /$ $\mathrm{mL}$ ) and incubated at $34^{\circ} \mathrm{C}$ for $30 \mathrm{~min}$ at low-speed rotation. Before sorting, the digested cells were stained with propidium iodide (PI, $1 \mu \mathrm{g} / \mu \mathrm{L}$ ). Then, the digested cells were filtered through a $40 \mu \mathrm{m}$ cellular filter and sorted by FACS, based on Hoechst 33342/PI staining, into two distinct channels. 


\section{Western blot analyses}

To detect the CEP70 protein, testes of Cep $70^{-1-}$ and WT male mice were pulverized in liquid nitrogen and collected in $500 \mu \mathrm{L}$ ice-cold Radio Immunoprecipitation Assay Lysis Buffer (RIPA) buffer containing protease inhibitors. After that, the lysates were diluted with $2 \times$ Laemmli sample buffer (Bio-Rad, 1610737), and boiled in water for $10 \mathrm{~min}$. The protein samples were separated by Sodium Dodecyl Sulfate-Polyacrylamide Gel Electrophoresis (SDS-PAGE, 10\% acrylamide running gel) and then electrically transferred to polyvinylidene fluoride (PVDF) membranes. After transfer, the PVDF membranes were blocked with 5\% skimmed milk in Tris-buffered saline $(10 \mathrm{mM}$ Tris, $150 \mathrm{mM} \mathrm{NaCl}, \mathrm{pH} 7.5)$ containing $0.1 \%$ Tween-20 (TBST) for $1 \mathrm{~h}$ at room temperature and then immunoblotted at $4{ }^{\circ} \mathrm{C}$ overnight with Anti-CEP70 antibody (Abcam, ab227456, 1:1000, Rabbit) and Anti$\beta$-actin mouse monoclonal antibody (TransGen Biotech, HC201, 1:1000). After washing in TBST, the membranes were incubated for $1 \mathrm{~h}$ with Anti-rabbit or mouse HRPconjugated secondary antibody (1:3000). Finally, protein bands were visualized by an enhanced chemiluminescence detection system (Tanon-5200). Western blot images were processed using ImageJ software (Wayne Rasband, USA).

\section{Sperm motility assays}

Sperm motility measurements were performed as previously described ${ }^{64}$. Briefly, the cauda epididymis was dissected from an adult mouse and cut into pieces in $1 \mathrm{~mL}$ of human tubal fluid (HTF), and exuded using the "swimout" method at $37^{\circ} \mathrm{C}$ for $30 \mathrm{~min}$ to collect sperm. Then, $10 \mu \mathrm{L}$ of the exudate was put into a glass cell chamber (Leja Products BV, Nieuw-Vennep, The Netherlands) and kept on a heating platform at $37^{\circ} \mathrm{C}$. The spermatozoa was further observed through a $\times 20$ objective lens (Olympus BX51 microscope). A CCD camera (Olympus) was used to image the observation area of each chamber. Spermatozoa samples were analyzed using computer-aided sperm analysis (CASA, CEROS v.12, Hamilton Thorne Research), which were implemented using Minitube sperm visual digital semen assessment system (12500/ 1300, Minitube Group, Tiefenbach, Germany). The total number of sperm, motile sperm and the proportion of progressive sperm were analyzed.

\section{Scanning and transmission electron microscopy}

For scanning electron microscopy (SEM), epididymal sperm of WT and Cep $70^{-1-}$ male mice (10-weeks old) were collected and shredded in HTF medium using the "swim-out" method at $37^{\circ} \mathrm{C}$, shaken every $10 \mathrm{~min}$ three times. Then, the supernatant containing sperm was placed in a clean centrifuge tube and pelleted at $300 \times g$ for $5 \mathrm{~min}$. After that, the sperm was resuspended in an electron microscopy fixative (Servicebio, G1102), fixed for $2 \mathrm{~h}$ at room temperature, and transferred to $4{ }^{\circ} \mathrm{C}$ for preservation. Sperm samples were blocked with $1 \%$ OsO4 in $0.1 \mathrm{M}$ PBS $(\mathrm{pH}=7.4)$ for $1-2 \mathrm{~h}$ at room temperature. After washing in PBS three times (15 min each), the sperm samples were gradually dehydrated in increasing concentrations of ethanol $(30 \%, 50 \%, 70 \%, 80 \%, 90 \%, 95 \%$, and $100 \%$ twice; 15 min each) and in isoamyl acetate (Sinaopharm Group Chemical Reagent Company, 10003128) for $15 \mathrm{~min}$. After that, the samples were dried with a critical point dryer (Quorum, K850). Specimens were attached to metallic stubs using carbon stickers and sputter-coated with gold for $30 \mathrm{~s}$ using a Lon sputtering apparatus (Hitachi, MC1000). Finally, the samples were imaged using a scanning electron microscope (Hitachi, SU8100) by the Wuhan Servicebio Technology Company.

For transmission electron microscopy (TEM), the size of testes should be no more than $1 \mathrm{~mm}^{3}$. The testes samples were fixed in an electron microscopy fixative at room temperature for $1 \mathrm{~h}$ and then transferred into an Eppendorf (EP) tube with fresh TEM fixative for further fixation. Then, the tissues were washed with 0.1 M PBS (pH 7.4) three times for $15 \mathrm{~min}$ each. The tissues were fixed with $1 \% \mathrm{OsO} 4$ in PBS for $2 \mathrm{~h}$ at room temperature, and then rinsed in PBS three times for $15 \mathrm{~min}$ each. The samples were gradually dehydrated in increasing concentrations of ethanol (30\%, 50\%, 70\%, 80\%, 95\%, and 100\% twice; $20 \mathrm{~min}$ each) and in $2 \hat{\mathrm{A}}$ acetone (Sinaopharm Group Chemical Reagent Co. LTD, 10000418) for $15 \mathrm{~min}$. The samples were then infiltrated and embedded in resin according to the following steps: acetone: EMBed 812 $($ SPI, $90529-77-4)=1: 1$ for $2-4 \mathrm{~h}$ at $37^{\circ} \mathrm{C}$; acetone: EMBed $812=1: 2$ overnight at $37^{\circ} \mathrm{C}$; and pure EMBed 812 for $5-8 \mathrm{~h}$ at $37^{\circ} \mathrm{C}$. After that, pure EMBed 812 was poured into the embedding model, the tissues were inserted into the pure EMBed 812, and then kept at $37^{\circ} \mathrm{C}$ overnight. The embedding models with resin and samples were moved into a $65^{\circ} \mathrm{C}$ oven to polymerize for more than $48 \mathrm{~h}$. Then, the resin blocks were removed from the embedding models for standby application at room temperature. The resin blocks were cut to $60-80 \mathrm{~nm}$ thickness using an ultra-microtome (Leica, Leica UC7), and the tissues were fished onto 150 mesh cuprum grids with formvar film. The copper mesh was stained in the dark with $2 \%$ uranium acetate saturated alcohol solution for $8 \mathrm{~min}$, rinsed in $70 \%$ ethanol three times, and then rinsed in ultrapure water three times, $2.6 \%$ lead citrate was used to avoid $\mathrm{CO} 2$ staining for $8 \mathrm{~min}$, and then rinsed with ultrapure water three times. After drying with filter paper, the cuprum grids were placed into the grid board and dried overnight at room temperature. The cuprum grids were observed under a transmission electron microscope (Hitachi, HT7800) and images were taken by the Wuhan Servicebio Technology Company. 


\section{TUNEL assays}

Terminal deoxynucleotidyl transferase dUTP nick end labeling (TUNEL) assay was carried out according to the manufacturer instructions of In Situ Cell Death Detection Kit (Roche, 11684817910). Images were captured using a laser scanning confocal microscope (3D HISTECH, Pannoramic MIDI).

\section{Proteomics analysis of testes of WT and Cep70 $70^{-1-}$ male mice}

After protein extraction and trypsin digestion of testis of WT $(n=3)$ and Cep $70^{-1-}(n=3)$ male mice, the peptide was desalted by Strata X C18 SPE column (Phenomenex) and vacuum-dried. The peptide was reconstituted in $0.5 \mathrm{M}$ TEAB and processed according to the manufacturer protocol for tandem mass tag (TMT) kit/isobaric tags for relative and absolute quantitation (iTRAQ) kit. Briefly, one unit of TMT/iTRAQ reagent were thawed and reconstituted in acetonitrile. The peptide mixtures were then incubated for $2 \mathrm{~h}$ at room temperature and pooled, desalted, and dried by vacuum centrifugation. After TMT/ iTRAQ labeling, the tryptic peptides were fractionated into fractions by high $\mathrm{pH}$ reverse-phase high-performance liquid chromatography (HPLC) using Thermo Betasil C18 column ( $5 \mu \mathrm{m}$ particles, $10 \mathrm{~mm}$ ID, $250 \mathrm{~mm}$ length). After HPLC fractionation, the tryptic peptides were analyzed by LC-tandem mass spectrometry (MS/MS). The resulting MS/MS data were processed using Maxquant search engine (v.1.5.2.8). False discovery rate (FDR) was adjusted to $<1 \%$ and minimum score for modified peptides was set $>40$.

According to the data quality control and search, a Gene Ontology (GO) annotation proteome was derived from the UniProt-GOA database (http://www.ebi.ac.uk/ GOA/). Then proteins were classified by GO annotation based on three categories: biological process, cellular component and molecular function. Identified proteins domain functional description were annotated by InterProScan (a sequence analysis application, http://www.ebi. ac.uk/interpro/) based on protein sequence alignment method, and the InterPro domain database was used. The Kyoto Encyclopedia of Genes and Genomes (KEGG) database was used to annotate protein pathway. For functional enrichment of GO, pathway, and protein domain, a two-tailed Fisher's exact test of was employed to test the enrichment of the differentially expressed proteins (DEPs) against all identified proteins based on GO annotation, the KEGG database, and the Interpro domain database. A corrected $p$-value $<0.05$ was considered as significant.

Further hierarchical clustering was based on DEP functional classifications (such as GO, domain, pathway, and complex). We first collated all the categories obtained after enrichment along with their $p$-values, and then filtered for those categories which were at least enriched in one of the clusters with a $p$-value $<0.05$. This filtered $p$ value matrix was transformed by the function $x=-\log 10$ ( $p$-value). Finally, the $x$ values were $z$-transformed for each functional category. These $z$-scores were then clustered by one-way hierarchical clustering (Euclidean distance and average linkage clustering) in Genesis. Cluster membership was visualized by a heatmap using the "heatmap.2" function from the "gplots" R-package.

Protein-protein interaction (PPI) networks of all the DEPs, database accession, or sequence, were searched against the STRING database (version 10.1). Only interactions between the proteins belonging to the searched data set were selected, thereby excluding external candidates. STRING defines a metric called "confidence score" to define interaction confidence; we fetched all interactions that had a confidence score $\geq 0.7$ (high confidence). The interaction network from STRING was visualized in the R-package "networkD3." The TMT proteomics analysis in our research was supported by Jingjie PTM BioLabs.

\section{Parallel reaction monitoring (PRM)}

The trypsin-digested peptides of WT and Cep $70^{-1-}$ male mice testes were dissolved in an aqueous solution containing $0.1 \%$ formic acid and $2 \%$ acetonitrile and then subjected to gradient treatment with an aqueous solution containing $0.1 \%$ formic acid and $90 \%$ acetonitrile, all at a constant flow rate of $500 \mathrm{~nL} / \mathrm{min}$ on an EASY-nLC 1000 ultra-performance liquid chromatography (UPLC) system (ThermoFisher Scientific). After treatment, the peptides were subjected to a Nanospray ionization (NSI) source followed by MS/MS in a Q ExactiveTM Plus (ThermoFisher Scientific) coupled online to the UPLC. The ion source voltage was set to $2.1 \mathrm{kV}$, and the peptide precursor ions and their secondary fragments were detected and analyzed by high-resolution Orbitrap. The $m / z$ scan range was $350-1000$ for the full MS at a resolution of $\times 70,000$, and peptides were then selected for MS/MS using normalized collision energy (NCE) setting of 27, and the fragments were detected in the Orbitrap at a resolution of $\times 17,500$. The data acquisition mode uses the dataindependent acquisition (DIA) program that alternated between one MS scan followed by 20 PRM scans, and the fragmentation energy of the higher energy collisional dissociation (HCD) was set to 27. The target of automatic gain control (AGC) and maximum injection time (Max IT) for full MS and MS/MS were set at 3E6/50 ms and $1 \mathrm{E} 5 / 200 \mathrm{~ms}$, respectively. The isolation window for MS/ MS was set at $1.6 \mathrm{~m} / \mathrm{z}$. PRM data were manually curated within Skyline (version 3.6) ${ }^{65}$. The peptide parameters were set as follows: enzyme was set as Trypsin [KR/P], and max missed cleavage was set as 2 and the peptide length was set as $7-25$ amino acid residues, and variable 
modification was set as alkylation on cysteine. The transition parameters were set as follows: precursor charges were set as 2, 3, ion charges were set as 1 , ion types were set $\mathrm{as} \mathrm{b}, \mathrm{y}$. The product ions were set from ion 3 to last ion, the ion match tolerance was set as $0.02 \mathrm{Da}$.

\section{Statistical analysis}

Data are presented as the mean \pm standard deviation (SD) of at least three independent replicates, and error bars indicate SD. Statistical analyses were performed using a two-tailed Student's $t$-test $(n \geq 3)$. Differences were considered significant at $\left.p<0.05\left(^{*}\right), p<0.01{ }^{(* *}\right)$, and $p<0.001{ }^{(* * * *)}$.

\section{Acknowledgements}

We are grateful to Prof. Hengyu Fan (Zhejiang University) for providing the rat monoclonal antibody against SYCP3 and Prof. Wei Li (Institute of zoology, Chinese Academy of Sciences) for the guidance on CASA analysis, and all the patients who provided samples to this research. This work was supported by the National Natural Science Foundation of China (31890781, 31900604, 81730038 and 81521002).

\section{Author details \\ 'Center for Reproductive Medicine, Department of Obstetrics and Gynecology, Peking University Third Hospital, Beijing, China. ${ }^{2}$ National Clinical Research Center for Obstetrics and Gynecology (Peking University Third Hospital), Beijing, China. ${ }^{3}$ Key Laboratory of Assisted Reproduction (Peking University), Ministry of Education, Beijing, China. ${ }^{4}$ Beijing Key Laboratory of Reproductive Endocrinology and Assisted Reproductive Technology, Beijing, China. ${ }^{5}$ Research Units of Comprehensive Diagnosis and Treatment of Oocyte Maturation Arrest, Chinese Academy of Medical Sciences, Beijing, China}

\section{Author contributions}

Q.L. and L.Y. designed experiments, collected data, and wrote the manuscript. Q.G, X.C., and N.W. performed the animal experiments and carried out the flow cytometric analysis and the cell sorting assay. S.S. and AD.S. are responsible for analyzing the proteomic data. J.Q. and L.Y. supervised the study and the manuscript writing. All authors read and approved the final paper.

\section{Data availability}

All data, including its supplementary information files, supporting the findings of this study are included in this published article. The mass spectrometry proteomics data have been deposited to the ProteomeXchange Consortium (http://proteomecentral.proteomexchange.org) via the iProX partner repository with the data set identifier PXD023680.

\section{Ethics approval}

Animal experimental procedures were followed as the Institutional Animal Welfare and Ethics Committee policies of Peking University (item number: LA2018261). The collection of human samples was approved in 2012 by the Reproductive Medicine Ethics Committee of Peking University Third Hospital (item number: 2012sz018). All the DNA samples of clinical azoospermia patients used in this project have obtained informed consent before starting.

\section{Conflict of interest}

The authors declare no competing interests.

\section{Publisher's note}

Springer Nature remains neutral with regard to jurisdictional claims in published maps and institutional affiliations.

Supplementary information The online version contains supplementary material available at https://doi.org/10.1038/s41419-021-03755-z.
Received: 8 March 2021 Revised: 19 April 2021 Accepted: 20 April 2021 Published online: 12 May 2021

\section{References}

1. Luk, B. H. \& Loke, A. Y. A review of supportive interventions targeting individuals or couples undergoing infertility treatment: directions for the development of interventions. J. Sex Marital Ther. 42, 515-533 (2016).

2. Esteves, S. C. \& Chan, P. A systematic review of recent clinical practice guidelines and best practice statements for the evaluation of the infertile male. Int. Urol. Nephrol. 47, 1441-1456 (2015).

3. Babakhanzadeh, E., Nazari, M., Ghasemifar, S. \& Khodadadian, A. Some of the factors involved in male infertility: a prospective review. Int. J. Gen. Med. 13, 29-41 (2020).

4. Cannarella, R., Condorelli, R. A., Mongiò̀, L. M., La Vignera, S. \& Calogero, A. E. Molecular biology of spermatogenesis: novel targets of apparently idiopathic male infertility. Int. J. Mol. Sci. 21, 1728 (2020).

5. Tuttelmann, F., Ruckert, C. \& Ropke, A. Disorders of spermatogenesis: perspectives for novel genetic diagnostics after 20 years of unchanged routine. Med. Genet. 30, 12-20 (2018).

6. Matzuk, M. M. \& Lamb, D. J. The biology of infertility: research advances and clinical challenges. Nat. Med. 14, 1197-1213 (2008).

7. Kanatsu-Shinohara, M. \& Shinohara, T. Spermatogonial stem cell self-renewal and development. Annu. Rev. Cell Dev. Biol. 29, 163-187 (2013).

8. $\mathrm{Xu}, \mathrm{Z}$. et al. CIB4 is essential for the haploid phase of spermatogenesis in micet. Biol. Reprod. 103, 235-243 (2020).

9. Oakberg, E. F. Duration of spermatogenesis in the mouse and timing of stages of the cycle of the seminiferous epithelium. Am. J. Anat. 99, 507-516 (1956).

10. Hermo, L., Pelletier, R. M., Cyr, D. G. \& Smith, C. E. Surfing the wave, cycle, life history, and genes/proteins expressed by testicular germ cells. Part 2: Changes in spermatid organelles associated with development of spermatozoa. Microsc. Res. Tech. 73, 279-319 (2010).

11. de Kretser, D. M., Loveland, K. L., Meinhardt, A., Simorangkir, D. \& Wreford, N. Spermatogenesis. Hum. Reprod. 13, 1-8 (1998).

12. Jan, S. Z. et al. Unraveling transcriptome dynamics in human spermatogenesis. Development 144, 3659-3673 (2017).

13. Wang, M. et al. Single-cell RNA sequencing analysis reveals sequential cell fate transition during human spermatogenesis. Cell Stem Cell 23, 599-614 e594 (2018).

14. Amaral, A. et al. Human sperm tail proteome suggests new endogenous metabolic pathways. Mol. Cell Proteom. 12, 330-342 (2013).

15. Andersen, J. S. et al. Proteomic characterization of the human centrosome by protein correlation profiling. Nature 426, 570-574 (2003).

16. Shi, X. et al. CEP70 Protein interacts with ?-tubulin to localize at the centrosome and is critical for mitotic spindle assembly. J. Biol. Chem. 286, 33401-33408 (2011)

17. Sha, Y. W. et al. A homozygous CEP135 mutation is associated with multiple morphological abnormalities of the sperm flagella (MMAF). Gene $\mathbf{6 3 3}, 48-53$ (2017).

18. Hall, E. A. et al. Acute versus chronic loss of mammalian Azi1/Cep131 results in distinct ciliary phenotypes. PLoS Genet. 9, e1003928 (2013).

19. Shi, $\mathbf{X}$. et al. Cep70 promotes microtubule assembly in vitro by increasing microtubule elongation. Acta Biochim. Biophys. Sin. 44, 450-454 (2012).

20. Shi, $X$. et al. Cep70 regulates microtubule stability by interacting with HDAC6 FEBS Lett. 589, 1771-1777 (2015).

21. Wilkinson, C. J., Carl, M. \& Harris, W. A. Cep70 and Cep131 contribute to ciliogenesis in zebrafish embryos. BMC Cell Biol. 10, 17 (2009).

22. Chen, Y. et al. Single-cell RNA-seq uncovers dynamic processes and critical regulators in mouse spermatogenesis. Cell Res. 28, 879-896 (2018).

23. Lei, W. L., Han, F., Hu, M. W., Liang, Q. X. \& Sun, Q. Y. Protein phosphatase 6 is a key factor regulating spermatogenesis. Cell Death Differ 27, 1952-1964 (2020).

24. Wu, S., Yan, M., Ge, R. \& Cheng, C. Y. Crosstalk between sertoli and germ cells in male fertility. Trends Mol. Med. 26, 215-231 (2020).

25. Fang, $X$. et al. Proteomics and single-cell RNA analysis of Akap4-knockout mice model confirm indispensable role of Akap4 in spermatogenesis. Dev. Biol. 454, 118-127 (2019).

26. Roy, A., Lin, Y. N., Agno, J. E., Demayo, F. J. \& Matzuk, M. M. Absence of tektin 4 causes asthenozoospermia and subfertility in male mice. FASEB J. 21, 1013-1025 (2007) 
27. Yang, K., Grzmil, P., Meinhardt, A. \& Hoyer-Fender, S. Haplo-deficiency of ODF1/ HSPB10 in mouse sperm causes relaxation of head-to-tail linkage. Reproduction 148, 499 (2014)

28. Li, Y. F. et al. CABYR binds to AKAP3 and Ropporin in the human sperm fibrous sheath. Asian J. Androl. 13, 266-274 (2011).

29. Fiedler, S. E., Dudiki, T., Vijayaraghavan, S. \& Carr, D. W. Loss of R2D2 proteins ROPN1 and ROPN1L causes defects in murine sperm motility, phosphorylation, and fibrous sheath integrity. Biol. Reprod. 88, 41 (2013).

30. Yang, Y., Richard, O. \& Antonio, M. V. Developmental expression of spermatidspecific thioredoxin-1 protein: transient association to the longitudinal columns of the fibrous sheath during sperm tail formation. Biol. Reprod. 67, 1546-1554 (2002).

31. Smith, T. B., Baker, M. A., Connaughton, H. S., Habenicht, U. \& Aitken, R. J. Functional deletion of Txndc2 and Txndc3 increases the susceptibility of spermatozoa to age-related oxidative stress. Free Radic. Biol. Med. 65, 872-881 (2013).

32. Fujihara, Y. et al. Identification of multiple male reproductive tract-specific proteins that regulate sperm migration through the oviduct in mice. Proc. Natl Acad. Sci. USA 116, 201908736 (2019).

33. Xu, K., Yang, L., Zhang, L. \& Qi, H. Lack of AKAP3 disrupts integrity of the subcellular structure and proteome of mouse sperm and causes male sterility. Development 147, dev181057 (2020).

34. Buffone, M. G. et al. Recombinant mouse sperm ZP3-binding protein (ZP3R/ sp56) forms a high order oligomer that binds eggs and inhibits mouse fertilization in vitro. J. Biol. Chem. 283, 12438 (2008).

35. Jiang, S. et al. Fluoride exposure arrests the acrosome formation during spermatogenesis via down-regulated Zpbp1, Spaca1 and Dpy1912 expression in rat testes. Chemosphere 226, 874-882 (2019).

36. Yoshinori, K. et al. Biogenesis of sperm acrosome is regulated by pre-mRNA alternative splicing of Acrbp in the mouse. Proc. Natl Acad. Sci. USA 113, E3696-E3705 (2016).

37. Jiang, S. et al. Fluoride exposure arrests the acrosome formation during spermatogenesis via down-regulated Zpbp1, Spaca1 and Dpy1912 expression in rat testes. Chemosphere 226, 874-882 (2019).

38. Chen, S. R. et al. The control of male fertility by spermatid-specific factors: searching for contraceptive targets from spermatozoon's head to tail. Cell Death Dis. 7, e2472 (2016).

39. Satouh, Y., Inoue, N., Ikawa, M. \& Okabe, M. Visualization of the moment of mouse sperm-egg fusion and dynamic localization of IZUMO1. J. Cell Sci. 125, 4985-4990 (2012)

40. Zhou, C., Huang, L., Shi, D. S. \& Jiang, J. R. Effects of latrunculin A on the relocation of sperm IZUMO1 during gamete interaction in mouse. Mol. Reprod. Dev. 84, 1183-1190 (2017).

41. Mendoza-Lujambio, I. The Hook1 gene is non-functional in the abnormal spermatozoon head shape (azh) mutant mouse. Hum. Mol. Genet. 11, 1647-1658 (2002).

42. Donkor, F. F. Outer dense fibre protein 2 (ODF2) is a self-interacting centrosomal protein with affinity for microtubules. J. Cell Sci. 117, 4643 (2004).

43. Bourmaud, A., Gallien, S. \& Domon, B. Parallel reaction monitoring using quadrupole-orbitrap mass spectrometer: principle and applications. Proteomics 16, 2146-2159 (2016)

44. O'Donnell, L., Nicholls, P. K., O’Bryan, M. K., McLachlan, R. I. \& Stanton, P. G. Spermiation: the process of sperm release. Spermatogenesis 1, 14-35 (2011).
45. Jurado, S. et al. The Zinc-finger protein ASCIZ regulates B cell development via DYNLL1 and Bim. J. Exp. Med. 209, 1629-1639 (2012).

46. King, A. et al. Dynein light chain regulates adaptive and innate B cell development by distinctive genetic mechanisms. PLoS Genet. 13, e1007010 (2017).

47. Jungwirth, A. et al. European Association of Urology guidelines on male infertility: the 2012 update. Eur. Urol. 62, 324-332 (2012).

48. Sha, Y. W. et al. TDRD6 is associated with oligoasthenoteratozoospermia by sequencing the patient from a consanguineous family. Gene 659, 84-88 (2018).

49. O'Donnell, L., McLachlan, R. I., Merriner, D. J., O'Bryan, M. K. \& Jamsai, D. KATNB1 in the human testis and its genetic variants in fertile and oligoasthenoteratozoospermic infertile men. Andrology 2, 884-891 (2014).

50. Nasirshalal, M. et al. Identification of the PRM1 gene mutations in oligoasthenoteratozoospermic men. Andrologia 52, e13872 (2020).

51. Crapster, J. A., Rack, P. G., Hellmann, Z. J., Le, A. D. \& Chen, J. K. HIPK4 is essential for murine spermiogenesis. elife 9, e50209 (2020).

52. Kim, K.-S. Mouse sperm protein sp56 is a component of the acrosomal matrix. Biol. Reprod. 64, 36 (2001).

53. Inoue, N., Ikawa, M., Isotani, A. \& Okabe, M. The immunoglobulin superfamily protein Izumo is required for sperm to fuse with eggs. Nature 434, 234-238 (2005).

54. Fujihara, Y. et al. SPACA1-deficient male mice are infertile with abnormally shaped sperm heads reminiscent of globozoospermia. Development 139, 3583-3589 (2012)

55. Shen, Y. et al. Loss-of-function mutations in QRICH2 cause male infertility with multiple morphological abnormalities of the sperm flagella. Nat. Commun. 10 433 (2019).

56. Li, Y. F. et al. CABYR isoforms expressed in late steps of spermiogenesis bind with AKAPs and ropporin in mouse sperm fibrous sheath. Reprod. Biol. Endocrinol. 8, 101 (2010).

57. Yuan, S. et al. Spata6 is required for normal assembly of the sperm connecting piece and tight head-tail conjunction. Proc. Natl Acad. Sci. USA 112, E430-E439 (2015).

58. Wang, X., Wei, Y., Fu, G., Li, H. \& Yu, L. Tssk4 is essential for maintaining the structural integrity of sperm flagellum. Mol. Hum. Reprod. 21, 136 (2015).

59. $\mathrm{Xu}, \mathrm{B}$. et al. Targeted deletion of Tssk1 and 2 causes male infertility due to haploinsufficiency. Dev. Biol. 319, 211-222 (2008).

60. Geyer, C. B. et al. A missense mutation in the Capza3 gene and disruption of Factin organization in spermatids of repro32 infertile male mice. Dev. Biol. 330, 142-152 (2009).

61. Wang, $\mathrm{H}$. et al. One-step generation of mice carrying mutations in multiple genes by CRISPR/Cas-mediated genome engineering. Cell 153, 910-918 (2013).

62. Sha, Q. Q. et al. CNOT6L couples the selective degradation of maternal transcripts to meiotic cell cycle progression in mouse oocyte. EMBO J. 37, e99333 (2018).

63. Jiang, Y., Zhang, H. Y., Lin, Z., Zhu, Y. Z. \& Fan, H. Y. CXXC finger protein 1mediated histone $\mathrm{H} 3$ lysine-4 trimethylation is essential for proper meiotic crossover formation in mice. Development 147, dev.183764 (2020).

64. Liu, C. et al. Sirt1 regulates acrosome biogenesis by modulating autophagic flux during spermiogenesis in mice. Development 144, 441-451 (2017).

65. MacLean, B. et al. Skyline: an open source document editor for creating and analyzing targeted proteomics experiments. Bioinformatics 26, 966-968 (2010). 